\title{
An official European Respiratory Society/ American Thoracic Society technical standard: field walking tests in chronic respiratory disease
}

\author{
Anne E. Holland, Martijn A. Spruit, Thierry Troosters, Milo A. Puhan, \\ Véronique Pepin, Didier Saey, Meredith C. McCormack, Brian W. Carlin, \\ Frank C. Sciurba, Fabio Pitta, Jack Wanger, Neil Maclntyre, David A. Kaminsky, \\ Bruce H. Culver, Susan M. Revill, Nidia A. Hernandes, Vasileios Andrianopoulos, \\ Carlos Augusto Camillo, Katy E. Mitchell, Annemarie L. Lee, Catherine J. Hill and \\ Sally J. Singh
}

Affiliations: For a full list of the authors' affiliations please refer to the Acknowledgements.

Correspondence: Anne E. Holland, La Trobe University Clinical School, Alfred Health, Level 4, The Alfred Centre, 99 Commercial Rd, Melbourne 3004, Australia. E-mail: a.hollanddalfred.org.au

ABSTRACT Field walking tests are commonly employed to evaluate exercise capacity, assess prognosis and evaluate treatment response in chronic respiratory diseases. In recent years, there has been a wealth of new literature pertinent to the conduct of the 6-min walk test (6MWT), and a growing evidence base describing the incremental and endurance shuttle walk tests (ISWT and ESWT, respectively). The aim of this document is to describe the standard operating procedures for the 6MWT, ISWT and ESWT, which can be consistently employed by clinicians and researchers.

The Technical Standard was developed by a multidisciplinary and international group of clinicians and researchers with expertise in the application of field walking tests. The procedures are underpinned by a concurrent systematic review of literature relevant to measurement properties and test conduct in adults with chronic respiratory disease.

Current data confirm that the 6MWT, ISWT and ESWT are valid, reliable and responsive to change with some interventions. However, results are sensitive to small changes in methodology. It is important that two tests are conducted for the 6MWT and ISWT.

This Technical Standard for field walking tests reflects current evidence regarding procedures that should be used to achieve robust results.

@ERSpublications

Technical Standard document: standard operating procedures for the 6MWT, ISWT and ESWT in chronic respiratory disease http://ow.ly/Bq2B9

This article has supplementary material available from erj.ersjournals.com

Received: Aug 162014 | Accepted: Aug 312014 | First published online: Oct 302014

Support statement: The realisation of this Technical Standard would not have been possible without the financial support of the European Respiratory Society and American Thoracic Society. Sally J. Singh is supported by the National Institute for Health Research (NIHR) Collaboration for Leadership in Applied Health Research and Care East Midlands (CLAHRC EM), and work took place at the University Hospitals of Leicester NHS Trust. Support was also provided by the NIHR Leicester Respiratory Biomedical Research Unit. The views expressed are those of the authors and not necessarily those of the NHS, the NIHR or the Dept of Health.

Conflict of interest: Disclosures can be found alongside the online version of this article at erj.ersjournals.com

Copyright @ERS 2014 


\section{Overview}

The aim of this Technical Standard is to document the standard operating procedures for the 6-min walk test (6MWT), incremental shuttle walk test (ISWT) and endurance shuttle walk test (ESWT) in adults with chronic respiratory disease. The testing procedures were developed by a multinational and multidisciplinary group of experts in field exercise testing, informed by a systematic review of the measurement properties and interpretation of the 6MWT, ISWT and ESWT in adults with chronic respiratory disease [1].

The key findings of the Technical Standard are as follows.

1) The 6-min walking distance (6MWD), ISWT and ESWT demonstrate good construct validity. Strong relationships with measures of exercise performance and physical activity support their conceptualisation as tests of functional exercise performance.

2) A lower 6MWD is strongly associated with increased risk of hospitalisation and mortality in people with chronic respiratory disease, with a small number of studies suggesting a similar relationship for the ISWT.

3) The 6MWD, ISWT and ESWT exhibit good test-retest reliability; however, there is strong evidence of a learning effect for the 6MWT and ISWT. Two tests should be performed when the 6MWT or ISWT are used to measure change over time.

4) The 6MWD, ISWT and ESWT are responsive to treatment effects in people with chronic respiratory disease; however, most studies have evaluated responsiveness to rehabilitation and fewer data are available to confirm responsiveness to other interventions.

5) The 6MWD and ISWT elicit a peak oxygen uptake $\left(V^{\prime} \mathrm{O}_{2}\right.$ peak $)$ that is similar to that during a cardiopulmonary exercise test (CPET). As a result, the contraindications and precautions for field testing should be consistent with those used for a CPET.

6) The 6MWD is very sensitive to variations in methodology, including use of encouragement, provision of supplemental oxygen, changes in track layout and length, and use of wheeled walkers. These factors should be held constant on repeat testing.

7) The $6 \mathrm{MWD}$ is the primary outcome of the $6 \mathrm{MWT}$.

8) The lowest arterial oxygen saturation measured by pulse oximetry $\left(\mathrm{SpO}_{2}\right)$ recorded during a $6 \mathrm{MWT}$ has emerged as an important marker of disease severity and prognosis; however, it may not be consistent with end-test $\mathrm{SpO}_{2}$. Continuous pulse oximetry is recommended during the 6MWT, to ensure that the lowest $\mathrm{SpO}_{2}$ is recorded.

9) Available evidence suggests a minimal important difference (MID) of $30 \mathrm{~m}$ for the 6MWD in adults with chronic respiratory disease.

10) The primary outcome of the ISWT is distance, measured to the nearest $10 \mathrm{~m}$.

11) The primary outcome of the ESWT is time, although distance has also been reported.

12) Application of reference equations for $6 \mathrm{MWD}$ or ISWT to an individual gives rise to substantial variation in predicted values. If reference values are to be used, an equation generated and verified in a local population should be applied where possible.

13) Testing procedures that are consistent across the 6MWT, ISWT and ESWT include test location and staffing, patient assessment and preparation, use of oxygen and medications, indications for test cessation, and quality assurance. These procedures are detailed in the Technical Standard.

\section{Introduction}

The 6MWT plays a key role in evaluating functional exercise capacity, assessing prognosis and evaluating response to treatment across a wide range of respiratory diseases. Since publication of the previous American Thoracic Society (ATS) statement on the 6MWT in 2002 [2], new information regarding the $6 \mathrm{MWT}$ has emerged in a range of areas, including methods of test performance and interpretation. This increased body of knowledge has significant implications for the good conduct of the 6MWT in individuals with chronic respiratory disease, in both research and clinical settings.

The last 10 years have also seen a growing body of evidence describing the uses and measurement properties of the ISWT and the ESWT. Given the increasing uptake of these field tests in clinical practice, the scope of the 2002 document has been expanded to include the ISWT and ESWT. While the use of other field tests has also been reported in chronic respiratory disease (e.g. timed up and go, sit to stand test, 4-m gait speed test), the committee considered that there were currently insufficient data to include them in this version of the Technical Standard. 
The testing procedures outlined in this document are based on a systematic review of the literature describing the use and properties of these field walking tests in adults with chronic respiratory disease, including chronic obstructive pulmonary disease (COPD), interstitial lung disease (ILD), cystic fibrosis (CF), bronchiectasis, asthma, pulmonary arterial hypertension (PAH) and pulmonary vascular disease [1]. As a consequence, these standards are not intended for application to children or to individuals who do not have chronic respiratory disease. This Technical Standard provides guidance on the practical aspects of conducting field walking tests in a safe, effective and reproducible manner. This document is not intended to provide guidance regarding when or in which patients these tests should be performed. The measurement properties of each field walking test are described individually. Standard operating procedures common to all three tests are outlined and those specific to each test are described. A brief comparison of the three tests is included. Finally, important differences between this document and the previous ATS statement on the $6 \mathrm{MWT}$ are highlighted.

\section{Methods}

An ad hoc Task Force was assembled to develop technical standards for the performance of the 6MWT, ISWT and ESWT based upon a systematic review of the evidence [1]. Co-chairs were selected by the Pulmonary Rehabilitation Assembly and Proficiency Standards Committee of the ATS and the Rehabilitation and Chronic Care Assembly and Allied Respiratory Professionals Assembly of the European Respiratory Society (ERS), then approved by the leadership of both societies. Members of the Task Force were selected by the co-chairs on the basis of their expertise in application of field walking tests in research and/or clinical practice. All potential conflicts of interest were disclosed and managed according to the policies and procedures for joint ATS/ERS projects. Drafts of this document were prepared by two members (Anne E. Holland and Sally J. Singh), with all authors providing comment and suggestions for the final document. The methods checklist is presented in table 1 .

The systematic review which informs this document addressed seven questions relevant to the measurement properties, performance, clinical utility, reporting, monitoring, reference equations and interpretation for field walking tests in chronic respiratory disease. Full details are provided in a companion paper [1].

\section{The 6MWT: measurement properties important to test conduct}

The 6 MWT is a self-paced test of walking capacity. Patients are asked to walk as far as possible in 6 min along a flat corridor. The distance in metres is recorded. Standardised instructions and encouragement are commonly given during the test [2].

\section{Validity of 6MWD}

There is a large body of data demonstrating construct and criterion validity for the 6MWD in individuals with chronic respiratory disease [1]. Relationships are strongest with measures of maximal exercise

TABLE 1 Methods checklist

Yes

No

\section{Panel assembly}

Included experts for relevant clinical and nonclinical disciplines

Included individual who represents the views of patients and society at large

Included methodologist with appropriate expertise

\section{Literature review}

Performed in collaboration with a librarian

Searched multiple electronic databases

Reviewed reference lists of retrieved studies

\section{Evidence synthesis}

Applied pre-specified inclusion and exclusion criteria

Evaluated included studies for sources of bias

Explicitly summarised benefits and harms

Used PRISMA1 to report systematic review

Used GRADE to describe quality of evidence

Generation of recommendations

Used GRADE to rate the strength of recommendations $x$

$x$

$x$

$x$

$x$

$x$

$x$

$x$

PRISMA: Preferred Reporting Items for Systematic Reviews and Meta-Analyses; GRADE: Grading of Recommendations Assessment, Development and Evaluation; NA: not applicable. 
performance and physical activity (correlation coefficients 0.4-0.93). Relationships between 6MWD and respiratory function (forced expiratory volume in $1 \mathrm{~s}$ (FEV1), forced vital capacity and diffusing capacity of the lung for carbon monoxide (DLCO)) (correlation coefficients $0.31-0.55$ ) or health-related quality of life (HRQoL) (correlation coefficients $0.03-0.65$ ) are of weak to moderate strength across all disease groups examined.

Direct comparisons of the physiological demands of the 6MWT and CPET show that although measures of peak exercise performance ( $V^{\prime} \mathrm{O}_{2}$ peak and heart rate (HR) peak) are similar between the tests, the 6MWT has substantially lower ventilatory requirements (peak carbon dioxide production, peak ventilation and respiratory exchange ratio) [3-8], which may contribute to its tolerability in adults with chronic respiratory disease. Overall, these data lend support to the conceptualisation of the $6 \mathrm{MWT}$ as a test of functional exercise capacity.

\section{Reliability and learning effect for 6MWD}

The 6MWD is a reliable measure in people with chronic respiratory disease, with excellent intra-class correlation coefficients (ICCs) (range 0.82-0.99) [1]. There are no discernible differences in reliability across groups with different chronic respiratory diseases. Despite its excellent reliability, there is strong evidence of a learning effect for the 6MWD when two or more tests are conducted. 13 studies in patients with COPD show a pooled mean improvement on the second 6MWT of $26.3 \mathrm{~m}$ [1]. This estimate does not change when including only the subgroup of studies where the two tests are conducted within $24 \mathrm{~h}$ $(26.1 \mathrm{~m})$. The largest study to address this issue $(n=1514)$ reported a 95\% confidence interval for the learning effect of 24-29 m [9]. There is some variability across individuals: the proportion of participants who walked further on the second $6 \mathrm{MWT}$ ranged from $50 \%$ to $87 \%$ across studies. Fewer data are available in other chronic respiratory diseases, although the available studies tend to support a learning effect across all chronic respiratory diseases [1]. Whether the learning effect is equally significant for individuals who have previously performed multiple 6MWTs is difficult to establish, as few data are available. If the tests are repeated 3 months later, the learning effect appears to persist in individuals with COPD; however, it may be smaller in magnitude [10].

The effect of learning on the 6MWD is large enough to be clinically important when the 6MWT is used to evaluate response to treatment or change over time. In these situations, two 6MWTs should be performed and the best 6MWD recorded. Use of two 6MWDs may also decrease the sample size requirements for clinical trials, due to reduced variability in the pre- and post-intervention measures [11]. Where the 6MWD is used as a one-off measure to stage disease or assess risk (e.g. likelihood of hospitalisation or mortality), the magnitude of the learning effect may be less important and one test may be sufficient. However, clinicians should be mindful of the learning effect if the 6MWD is approaching pre-defined thresholds on which treatment decisions may be based $[12,13]$; in this situation, repeat testing should be considered. One test may also be sufficient for patients who have recently performed the test, where the learning effect is smaller (e.g. end of pulmonary rehabilitation) [10].

\section{Relationship of 6MWD to clinical outcomes}

The 6MWD has a strong relationship to important clinical outcomes in individuals with chronic respiratory disease. A shorter 6MWD was associated with increased mortality in 13 (93\%) out of the 14 COPD studies reviewed [1]. Associations were less consistent in ILD (four (50\%) out of eight studies) and PAH (six (66\%) out of nine studies) [1]. There is less evidence for the association of $6 \mathrm{MWD}$ with hospitalisation, but all studies in which it was evaluated ( $n=3$, COPD and ILD) found a statistically significant relationship [1]. It was outside the scope of this document to identify thresholds for 6MWD to categorise patients according to their risk for these outcomes.

\section{Methodological factors affecting test performance}

The 6MWD is highly sensitive to changes in methodology (table 2). Given the impact of encouragement on $6 \mathrm{MWD}$ [33] and the use of encouraged tests in the generation of reference equations [1], it is recommended that standardised phrases of encouragement are used (see later section on the testing protocol for 6MWT). Provision of supplemental oxygen [26-29], method for carrying the supplemental oxygen [31, 32] and use of wheeled walkers [20-25] also have an important impact on 6MWD. These factors must be kept constant on repeat testing. The $6 \mathrm{MWD}$ generated using an externally paced treadmill is substantially lower than in a hallway $[14,15]$, which is probably the result of the poor walking efficiency during treadmill walking in subjects unaccustomed to this activity. Externally paced treadmill testing is not recommended. Track layout and length may also affect performance on the test, especially when very short track lengths are used [19]; these factors should be kept constant where within-subject comparison of 6MWD on subsequent occasions 
TABLE 2 Effect of methodological variations on 6-min walking distance (6MWD)

\begin{tabular}{|c|c|c|c|}
\hline Variation in methodology & Studies $\mathbf{n}$ & First author [ref.] & Effect on 6MWD \\
\hline Hallway versus treadmill & 2 & $\begin{array}{l}\text { SteVens [14] } \\
\text { DE ALMEIDA [15] }\end{array}$ & $13-20 \%$ less on treadmill \\
\hline Indoors versus outdoors & 1 & BROOKS [16] & $4 \mathrm{~m}(1 \%)$ more outside \\
\hline Circular versus straight track & 2 & $\begin{array}{l}\text { BANSAL [17] } \\
\text { SCIURBA [18] }\end{array}$ & 13-19 m (3-5\%) more on circular track \\
\hline Track length & 2 & $\begin{array}{l}\text { SCIURBA [18] } \\
\text { BEEKMAN [19] }\end{array}$ & $\begin{array}{l}\text { No statistically significant difference in 6MWD } \\
\text { from tracks of } 15-121 \mathrm{~m} \\
50 \mathrm{~m} \text { more on } 30-\mathrm{m} \text { track compared to } 10-\mathrm{m} \text { track }\end{array}$ \\
\hline Wheeled walking aid versus no aid & 6 & $\begin{array}{c}\text { GuPTA [20] } \\
\text { HONEYMAN [21] } \\
\text { PROBSt [22] } \\
\text { RoOMI [23] } \\
\text { SOLWAY [24] } \\
\text { VAES [25] }\end{array}$ & $\begin{array}{c}\text { Weighted mean } 6.2 \% \text { more with wheeled walker } \\
\text { Range 2-46 m more with wheeled walker } \\
83 \mathrm{~m} \text { more with modern draisine compared } \\
\text { to wheeled walker }\end{array}$ \\
\hline With versus without oxygen & 4 & $\begin{array}{l}\text { DAVIDSON [26] } \\
\text { FUJIMOTO [27] } \\
\text { ROOYACKERS [28] } \\
\text { JOLLY [29] }\end{array}$ & 12-59 m more with oxygen \\
\hline Oxygen versus compressed air & 2 & $\begin{array}{c}\text { JOLLY [29] } \\
\text { MCDONALD [30] }\end{array}$ & 17-109 m more with oxygen \\
\hline $\begin{array}{l}\text { Carry oxygen versus oxygen in } \\
\text { wheeled cart }\end{array}$ & 1 & CRISAFULLI [31] & $23 \mathrm{~m}$ more with wheeled cart \\
\hline $\begin{array}{l}\text { Patient carries oxygen versus tester } \\
\text { carries oxygen }\end{array}$ & 1 & Woodcock [32] & $24 \mathrm{~m}$ versus $35 \mathrm{~m}$ improvement \\
\hline Encouragement & 1 & GUYATT [33] & $30.5 \mathrm{~m}$ more with encouragement \\
\hline Instructions & 1 & WEIR [34] & $\begin{array}{l}53 \text { m further when asked to walk as "fast" as } \\
\text { possible, rather than as "far" as possible }\end{array}$ \\
\hline
\end{tabular}

is required. For track lengths of $>15 \mathrm{~m}$, differences may be small enough such that tests on different track layouts can still be used for risk stratification [1]; however, more data are needed to confirm this.

\section{Monitoring and reporting for 6MWT}

Safety

Complications associated with the performance of the 6MWT are unusual. We were unable to find published reports of complications associated with performance of the 6MWT in clinical trials. Only two articles have specifically addressed the issue of complications during the 6MWT. In 741 patients attending an outpatient pulmonary rehabilitation programme who completed the $6 \mathrm{MWT}$ in accordance with a standardised protocol, including continuous monitoring of oxyhaemoglobin saturation $\left(\mathrm{SpO}_{2}\right)$ and $\mathrm{HR}$, adverse events were noted in $43(6 \%)$ of patients [35]. The most common adverse event was oxygen desaturation $<80 \%$, upon which the test was terminated by the operator (35 out of 43 ); chest pain (one out of 43) and tachycardia (one out of 43) were also recorded. No long-term adverse sequelae from these events were reported. In the remaining tests the patients developed intolerable symptoms and the test was discontinued, which would be expected during the $6 \mathrm{MWT}$ and is not considered a complication. A second study in ILD $(n=19)$ showed that desaturation to $<80 \%$ occurred in $58 \%$ of participants during the $6 \mathrm{MWT}$ [36]. Concurrent ECG monitoring showed no clinically significant arrhythmias that needed treatment, although atrial tachycardia occurred in one case.

\section{Measurements}

The 6MWD is the primary outcome of the 6MWT, given its excellent reliability and validity, as well as its strong relationship to important clinical outcomes. The $6 \mathrm{MWD}$ should be reported for every test in metres or feet. However, other outcomes have been reported, such as $\mathrm{SpO}_{2}$, HR responses, symptoms of dyspnoea and fatigue and 6 -min walk work $(6 \mathrm{MWD} \times$ body weight $)$.

\section{Oxyhaemoglobin saturation}

Oxygen desaturation during a 6MWT provides information regarding exercise-induced desaturation, disease severity and disease progress. Exercise-induced desaturation is associated with impaired daily 
physical activity, faster FEV1 decline and worse prognosis [37, 38], which supports its clinical importance. The 6MWT is more sensitive for identifying exercise-induced desaturation than cycle testing [39]. Measurements of $\mathrm{SpO}_{2}$ during the $6 \mathrm{MWT}$ are reliable [1], provided that an adequate pulse signal is obtained. However, change in $\mathrm{SpO}_{2}$ may be more variable in people with systemic sclerosis-associated ILD (SSc-ILD) than in other lung conditions (ICCs ranging 0.24-0.64 in SSc-ILD, compared to 0.80-0.97 in CF and COPD) [1], perhaps due to cutaneous involvement in SSc-ILD. The 6MWT may be safe without continuous monitoring of $\mathrm{SpO}_{2}$ [36]. However, constant monitoring of $\mathrm{SpO}_{2}$ during the 6MWT is needed to obtain an accurate measure of exercise-induced desaturation, as the lowest $\mathrm{SpO}_{2}$ often does not occur at the end of the test $[40,41]$.

A number of novel desaturation indices have been proposed in patients with ILD, with the aim of improving the ability of the 6MWT to predict mortality. These include the distance-saturation product [42-45], the desaturation area [46] and the desaturation-distance ratio [47]. As only a small number of studies are available, the utility of these measures has not been confirmed and they are not routinely collected during the $6 \mathrm{MWT}$.

\section{Heart rate}

The resting and end-test HR are often recorded during the 6MWT. Limited data are available regarding the reliability of HR measures during the 6MWT; however, change in HR recorded on the pulse oximeter appears reliable (ICCs $0.62-0.87$ ) [9, 48]. No studies have compared the HR obtained from pulse oximetry to that obtained with other methods (e.g. frequency meter). Other measures of HR response include HR recovery (HRR), which is the reduction in HR with rest that occurs after the 6MWT is concluded. A reduced HRR during the first minute after the $6 \mathrm{MWT}$ has been associated with poor outcomes, including increased mortality, in ILD [49] and PAH [50, 51]. However, there is not a universally accepted cut-off for HRR that is applicable across chronic respiratory diseases.

\section{Dyspnoea}

Dyspnoea may be an important determinant of the 6MWD in patients with chronic respiratory disease [52-55], where it reflects both the physiology of exercise limitation $[56,57]$ and the impact of exercise limitation on daily life [58]. Dyspnoea scores collected during the 6MWT exhibit good reliability, with the modified Borg dyspnoea scale [59] (ICCs 0.59-0.92) showing greater reliability than the 15-count dyspnoea scale (ICC 0.66) and visual analogue scale for dyspnoea (coefficient of variation 0.22) [1]; however, few studies have investigated these latter measures.

\section{Subjective fatigue}

Fatigue is a common feature in patients with chronic respiratory diseases, both as a local muscle phenomenon and general fatigue. This is reflected during the 6MWT, where COPD patients experienced more fatigue compared to healthy elderly subjects [60]. Patient-reported fatigue is commonly measured at the beginning and end of the 6MWT using the Borg scale [59], which exhibits moderate reliability [9, 61]. Fatigue measured during the 6MWT using the Borg scale is associated with lower 6MWT, slower gait speed, more severe airflow obstruction, more dyspnoea on exertion and lower HRQoL [1]. Notably, low-frequency muscle fatigue in the quadriceps is seldom found with the 6MWT, although low-frequency fatigue in distal leg muscles has been reported to be greater in COPD patients compared with controls [62].

\section{6-min walk work}

The 6-min walk work is the product of $6 \mathrm{MWD}$ and body weight, which may provide a better estimation of the work required to perform the test than distance alone. The $6 \mathrm{MWD} \times$ weight product correlates more strongly with DLCO and $V^{\prime} \mathrm{O}_{2}$ peak than 6MWD alone $[63,64]$. To date, no studies have investigated the sensitivity of $6 \mathrm{MWD} \times$ weight product to change over time. Additional studies are needed to better characterise the utility of the 6-min walk work in adults with respiratory disease.

In summary, the 6MWD is the primary outcome of the 6MWT and should be recorded on every test. The $\mathrm{SpO}_{2}$ and HR should be measured continuously during the $6 \mathrm{MWT}$, to ensure that the lowest $\mathrm{SpO}_{2}$ and the end-test HR are recorded. Care should be taken that obtaining these measures does not affect performance. Current data indicate that the 6MWT has an excellent safety profile when the test is stopped if $\mathrm{SpO}_{2}$ falls to $<80 \%$; few data are available to define the safety profile if desaturation to $<80 \%$ is permitted. Dyspnoea and subjective fatigue should be measured before and after the 6MWT. 


\section{Identifying meaningful change in 6MWD}

Minimal important difference for the 6MWD

Over the past 10 years, a number of new estimates of the MID for 6MWD have been published. Our systematic review [1] identified 11 studies, in COPD (six studies), ILD (three studies) and PAH (two studies). The majority of MID estimates from these studies are based on distribution-based methods (using statistical properties of the measure), rather than anchor-based methods (where change in 6MWD is related to another clinically important marker of change). Most values were generated for individuals with COPD participating in a rehabilitation programme rather than pharmacotherapy. The median estimate across all studies was $30 \mathrm{~m} \mathrm{[1]}$. There is currently little evidence to suggest that the MID varies according to patient characteristics, including the type of chronic lung disease or the baseline 6MWD. Most MID estimates were generated using group mean data and are best used to interpret group mean changes in 6MWD [65-67]. However, studies that derived the MID using methodologies that are applicable to individuals reported similar MID estimates $[68,69]$. More studies are required to explore the magnitude of important changes in $6 \mathrm{MWD}$ with different interventions and to establish the MID in other chronic respiratory diseases.

In summary, the available evidence suggests a MID of $30 \mathrm{~m}$ for adult patients with chronic respiratory disease. There is some variability across studies and methods to determine the MID; however, based on the large evidence base now available, we can be confident that the MID lies between 25 and $33 \mathrm{~m}$.

\section{Responsiveness of the 6MWD}

Few studies have been explicitly designed to assess the responsiveness of $6 \mathrm{MWD}$ to treatment effects, but a large number of randomised trials provide insights in this area. We chose the Cochrane reviews on exercise interventions for COPD [70, 71] and ILD [72] and the Cochrane review on endothelin receptor antagonists in PAH [73] to investigate the responsiveness of 6MWD to interventions of known efficacy. The metaanalyses for 6MWD showed highly statistically significant improvements in 6MWD, with effects between 34 and $78 \mathrm{~m}$ and effect sizes between 0.38 and 1.07 [1]. The standardised response means (mean change/ standard deviation of change) ranged from 0.2 to 1.2 [1]. The 6MWD therefore appears to be responsive to treatment effects in patients with COPD, ILD and PAH. It should be noted that most of these values have been generated from rehabilitation-based studies.

\section{Reference equations for 6MWD}

The systematic review identified 16 published studies from 1998 to 2013 that included 6MWD prediction equations from healthy adults [1]. One additional study has been published since the systematic review [74]. These studies were conducted using a wide variety of populations and methodologies. Track lengths ranged from 20 to $50 \mathrm{~m}$ and the number of tests ranged from one to four. Application of these equations to an individual gives rise to substantial variation in the predicted distance.

Factors that may affect the 6MWD in healthy adults, and therefore the predicted distance, include methodology for 6MWT, percentage of peak HR achieved, height, age, sex, weight and perhaps race/ ethnicity. The utility of \% predicted values in assessing clinically meaningful change in 6MWD over time has not been investigated.

Due to the wide variation in predicted 6MWD generated by different equations, reference equations generated and verified in a local population should be applied where possible. A summary of reference equations can be found in the accompanying systematic review [1].

\section{The ISWT and ESWT: measurement properties important to test conduct Validity of the ISWT and ESWT}

The ISWT is an externally paced maximal exercise test; the speed of walking is controlled by a series of pre-recorded signals. The speed of walking increases until the participant can no longer continue. The maximum duration of the test is $20 \mathrm{~min}$.

Given the progressive nature of the ISWT, a strong relationship with performance on laboratory-based tests might be anticipated. Seven studies in COPD and one in lung cancer show that the $V^{\prime} \mathrm{O}_{2}$ peak, estimated $V^{\prime} \mathrm{O}_{2}$ peak, peak work and distance measured on ISWT show moderate to strong correlations with measures of maximal exercise performance on CPET [1], with no difference in measured oxygen uptake $\left(V^{\prime} \mathrm{O}_{2}\right)$ between the tests $[6,75,76]$. Hill et al. [6] performed a minute-by-minute analysis, and both the ISWT and CPET demonstrated a linear response in $V^{\prime} \mathrm{O}_{2}$ peak, suggesting that the two tests provoke a similar cardiopulmonary response. In summary, the current literature suggests that the ISWT is a valid measure of cardiopulmonary exercise capacity in COPD and provokes a similar physiological response to a CPET. More evidence in other disease populations is required. 
The ESWT is a derivative of the ISWT, where patients walk for as long as possible at a predetermined percentage of maximum walking performance as assessed by the ISWT [77]. To set the speed for the ESWT, the ISWT must have been completed previously. The ESWT has been shown to elicit similar end-test HR and dyspnoea responses compared to a treadmill endurance test at the same intensity [77].

\section{Reliability and learning effect for ISWT}

Only a handful of studies have reported the reliability of the ISWT, with most data in COPD. One study documented an ICC of 0.88 (95\% CI 0.83-0.92) [78]. Another study reported an ICC of 0.89 [79]. These two studies suggest that the association between test-retest walk distances is strong, with the majority of variability being attributable to between-subject differences. More studies are needed to confirm these findings.

There is a small but statistically significant difference between the first two ISWTs performed (mean differences of 20 and $25 \mathrm{~m}$ for tests performed on the same day, and $23.5 \mathrm{~m}$ for tests performed on different days) $[6,80,81]$. The effect of learning on the ISWT is large enough to be clinically important when evaluating change over time. It is recommended that two ISWTs be performed and the best distance recorded. It remains to be established whether one test would be sufficient where the ISWT is used as a oneoff measure to stage disease or assess risk (e.g. likelihood of hospitalisation or mortality). Whether one test is sufficient if the test has previously been conducted, similar to the 6MWT, is still unclear. It is common to repeat the test once after an intervention, but whether this underestimates the impact of the intervention remains to be established. The reliability of the test in chronic respiratory diseases other than COPD has not been explored.

\section{Reliability and learning effect for ESWT}

Three studies have examined the reliability of the ESWT, all in COPD [6, 81, 82]. The differences between tests repeated on the same day were generally small and statistically nonsignificant (pooled mean difference $+26 \mathrm{~s}$ ). Two tests do not appear to be necessary, although it is acknowledged that the number of studies is limited. Measurements of $\mathrm{HR}, \mathrm{SpO}_{2}$ and modified Borg dyspnoea scale appear to repeat well during the test [1] The reliability of the ESWT in other chronic respiratory diseases has not been examined.

\section{Relationship of ISWT and ESWT to clinical outcomes}

A small number of studies show that the ISWT is a significant predictor of survival and re-admission in people with COPD, with a lower distance predicting a greater risk of admission [83-85]. The relationship of ESWT to these outcomes is unknown.

\section{Methodological factors affecting ISWT and ESWT performance}

Unlike the 6MWT, the track for the ISWT and the ESWT is fixed, and alterations of the course have not been studied. The effect of encouragement has not been directly observed, but the external pacing of the tests may make it difficult to override the test protocol. The impact of supplemental oxygen is influenced by the mode of delivery. If patients are required to support a cylinder independently, oxygen supplementation confers little advantage in walking distance compared to air walking conditions [86], but if the oxygen (or heliox) is carried by a clinician/researcher, the benefit is magnified [87]. Accordingly, if supplemental oxygen is to be used during testing, the same approach should be taken for repeat testing, with the device and the flow rate remaining constant.

\section{Monitoring and reporting for ISWT and ESWT \\ Safety}

Adverse events have not been directly studied or reported in association with either the ISWT or the ESWT. We were unable to find any literature describing complications of conducting the shuttle tests. To date, there is no clear guidance on the value of monitoring $\mathrm{SpO}_{2}$ and the level to which this may fall in any individual has yet to be described. However, consensus suggests that the ISWT and the ESWT should be discontinued if $\mathrm{SpO}_{2}$ falls below $80 \%$. The test has been reported in patients with cardiac disease [88-90] with no adverse events; to date the test has not been used for patients with PAH.

\section{Measurements}

The primary outcome of the ISWT is distance, reported as an accumulation of 10-m lengths. The minimum distance is $0 \mathrm{~m}$ if patients fail to complete the first $10 \mathrm{~m}$, and the maximum is $1020 \mathrm{~m}$. The ESWT, like all endurance tests, is reported as time (minutes and seconds); although it can be expressed as distance completed, this is less commonly reported for endurance tests in the literature. The speed that the participant walks is chosen from 16 available speeds $\left(1.78-6.00 \mathrm{~km} \cdot \mathrm{h}^{-1}\right)$, with the speed calculated from the 
performance on the ISWT. Additional outcomes have been reported; these include $\mathrm{SpO}_{2}, \mathrm{HR}$ responses, dyspnoea, fatigue and the reason for terminating the test.

\section{Identifying meaningful change in ISWT and ESWT}

Minimal important difference for the ISWT

The MID for the ISWT has been described in one paper that was specifically designed to assess this threshold [91] and confirmed within a second paper [92]. Both studies used a similar approach, with a preference-based anchor method using rehabilitation as the intervention. The original paper described that a change of $47.5 \mathrm{~m}$ (approximately five shuttles) was associated with feeling "slightly better", while a change of $78.7 \mathrm{~m}$ (approximately eight shuttles) was associated with the next rating ("better"). The authors concluded that the MID for the ISWT was $47.5 \mathrm{~m}$. More studies are required, exploring different interventions and different approaches, to describe the MID in COPD. The MID for other chronic respiratory diseases has not been explored.

\section{Minimal important difference for the ESWT}

The MID for the ESWT in patients receiving pulmonary rehabilitation or bronchodilation has been estimated using a distribution-based approach and anchor-based method (change in ESWT time and distance was related to patients' perception of change from baseline) [93]. Bronchodilation data indicated that a change of $65 \mathrm{~s}(95 \%$ CI $45-85 \mathrm{~s})$ or $85 \mathrm{~m}$ (95\% CI 60-115 m), representing 13-15\% of baseline, was associated with a one-point change on the Likert rating scale and thus likely to be perceivable to patients. An estimate of the MID could not be obtained from the pulmonary rehabilitation data, although preliminary data suggest this is in the region of $180 \mathrm{~s}$ [93]. It is possible that the MID may be context or intervention specific, although to date this has not been shown for 6MWD.

\section{Responsiveness of the shuttle walk tests}

Despite the ISWT being employed in a number of studies, only eight studies were explicitly designed to evaluate the responsiveness to treatment of the ISWT and/or ESWT [86, 94-100]. These studies suggest that the ISWT and ESWT are both responsive. The standardised response means (mean change/standard deviation of change) range from 0.72 to 1.55 for the ISWT and from 0.52 to 1.27 for the ESWT [1].

\section{Reference equations for ISWT and ESWT}

Three papers have described reference values for the ISWT, two from South America [101, 102] and one from the UK [103]. There is a need for more data, including diversity in the country of origin. Existing data suggest that age, sex and body mass index are important variables. Data from a single centre in the UK [1] included measures of strength (quadriceps maximum voluntary contraction) and physical activity (Duke Physical Activity Status Index and a physical activity monitor), in an attempt to further explain the variability; however, these additions did not improve the reference equation.

To date there has been no attempt to identify reference equations for the ESWT.

\section{Selecting a field walking test}

It is beyond the scope of this Technical Standard to recommend one field test over another. The systematic review underpinning this document did not address the question of the relative merits of each test. However, some general considerations that may influence test selection are outlined here.

Field walking tests may be performed to identify the individual's exercise capacity (peak exercise capacity, functional exercise capacity or endurance), factors limiting exercise performance (dyspnoea, subjective fatigue, musculoskeletal limitations) and often their response to an intervention. The field tests can also be used to identify a threshold to predict survival and the likelihood of a hospital readmission, with a more extensive body of evidence reporting use of 6MWD rather than ISWT or ESWT for this purpose [1]. Prescription of exercise intensity for pulmonary rehabilitation, using the established principles of exercise training, may also be a consideration. The uses of the 6MWT and the ISWT/ESWT have been reported in all of these circumstances [1]. Practical considerations include whether sufficient space is available. If the test of choice is the 6MWT then the test should be conducted as recommended along a course at least $30 \mathrm{~m}$ in length; if this space is not available then consideration should be given to using the ISWT/ESWT. Both the $6 \mathrm{MWT}$ and ISWT require two tests to be performed prior to an intervention [1] and thus the time taken to conduct each test is comparable.

The 6MWT and ISWT/ESWT offer quite different protocols: the 6MWT is self-paced, and the shuttle tests are externally paced. Although both tests generally provoke a $V^{\prime} \mathrm{O}_{2}$ similar to that seen on a CPET $[6,75,104-106]$, the precise pattern of response may be different for the externally paced tests (ISWT/ESWT) compared to a self-paced test $(6 \mathrm{MWT})$. The response of the ISWT mirrors the physiological response 


\section{TABLE 3 Equipment required for conduct of field walking tests}

At least one chair, positioned at one end of the walking course

A validated scale to measure dyspnoea and subjective fatigue

Sphygmomanometer for blood pressure measurement

Pulse oximeter

Stopwatch

Pre-measured marks along the track/corridor

Access to oxygen and telephone in case of an emergency

An emergency plan

Portable supplemental oxygen if required by patient to perform exercise test

Clipboard with reporting sheet and pen

observed in an incremental laboratory-based test, with an incremental increase in $V^{\prime} \mathrm{O}_{2}$ over time, whereas the self-paced 6MWT shows a steady-state $V^{\prime} \mathrm{O}_{2}$ profile after the third minute $[3,75,104]$. Because of the similarity in response of the ISWT and CPET, prescribing an exercise regimen as a percentage of peak performance on a field walking test may be easier with the ISWT than the 6MWT, because of the incremental nature of the test. Responses to the ESWT show a significantly more rapid rise in $V^{\prime} \mathrm{O}_{2}$ and ventilation than during the 6MWT, but similar end-test values [95]. The $V^{\prime} \mathrm{O}_{2}$ peak on ESWT does not differ significantly when compared to the ISWT and 6MWT [6]. The physiological responses to each test are reported in more detail in the systematic review [1].

The comparative sensitivity of field walking tests has been examined in small studies that have looked at rehabilitation or bronchodilation as the intervention, predominantly in COPD. PEPIN et al. [95] observed an enhanced response in the ESWT compared to the 6MWT after an acute administration of a bronchodilator, with standardised response means of 0.66 and 0.42, respectively. Similarly, EATON et al. [98] reported that, after a course of pulmonary rehabilitation, the magnitude of the change was proportionally greater on the ESWT than the 6MWT (92\% versus 17\%). The ESWT has also proven to be more responsive than the 6MWT in a randomised trial of an exercise intervention for COPD [107]. These results suggest that the ESWT may be more sensitive to change than the $6 \mathrm{MWT}$, following bronchodilation or pulmonary rehabilitation.

Given the differing protocols, physiological patterns of response, measurement properties and circumstances in which field walking tests are applied, the choice of test should be carefully considered. Regardless of the test selected, robust results can only be obtained with careful attention to testing procedures as described in the following sections.

\section{Test procedures applicable to all field walking tests \\ Equipment}

The equipment required to conduct a field walking test is listed in table 3.

\section{Test location and staffing}

The test should be conducted along a quiet course, physiotherapy gym or dedicated exercise testing room. A comfortable temperature is important and air conditioning should be available if needed. Testing should be performed in a location where a rapid, appropriate response to an emergency is possible. The appropriate location of a crash cart should be determined by the physician supervising the facility. Supplies that must be available include oxygen, sublingual nitroglycerine and salbutamol (metered dose inhaler or nebuliser). A telephone or other means of calling for help should be available in case of emergency.

The assessor performing the test should be certified in cardiopulmonary resuscitation with a minimum of Basic Life Support certification. Training, experience and certification in related healthcare fields are also desirable. A certified individual should be readily available to respond if needed. Physicians are not required to be present during all tests. The physician ordering the test or a supervising laboratory physician may decide whether physician attendance at a specific test is required.

\section{Patient assessment}

Given that all three field-based exercise tests can elicit a $V^{\prime} \mathrm{O}_{2}$ peak and peak HR that are similar to the CPET [1], absolute and relative contraindications for exercise testing should be consistent with recommendations for maximal exercise testing (table 4) [108]. Patients with any of these findings should be referred to the physician ordering or supervising the test for individual clinical assessment and a decision about the conduct of the test. Stable exertional angina is not an absolute contraindication for field walking tests, but patients with these symptoms should perform the test after using their anti-angina medication, and rescue 
TABLE 4 Absolute and relative contraindications for field walking tests

$\begin{array}{ll}\text { Absolute } & \text { Relative }\end{array}$

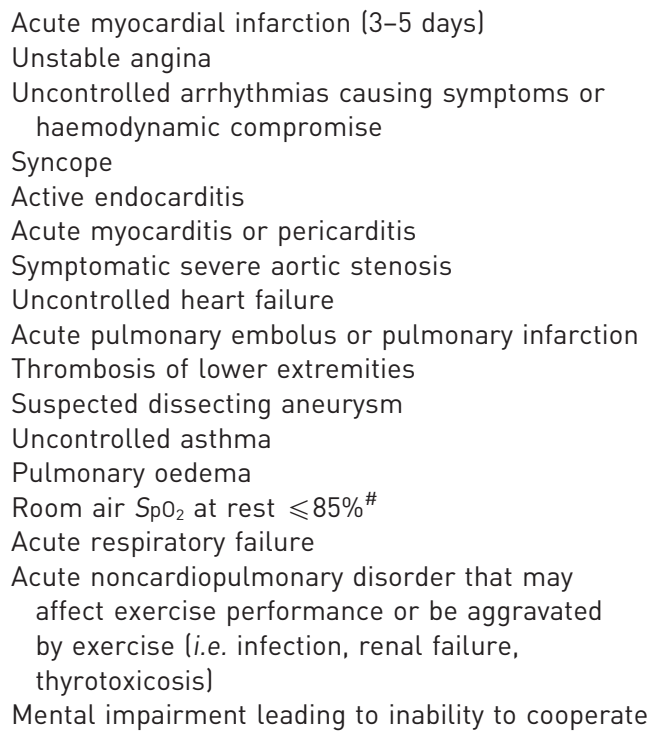

Left main coronary stenosis or its equivalent

Moderate stenotic valvular heart disease

Severe untreated arterial hypertension at rest

(200 mmHg systolic, $120 \mathrm{mmHg}$ diastolic)

Tachyarrhythmias or bradyarrhythmias

High-degree atrioventricular block

Hypertrophic cardiomyopathy

Significant pulmonary hypertension

Advanced or complicated pregnancy

Electrolyte abnormalities

Orthopaedic impairment that prevents walking

$\mathrm{SpO}_{2}$ : arterial oxygen saturation measured by pulse oximetry. ${ }^{\#}$ : exercise patient with supplemental oxygen. Reproduced from [108] with permission from the publisher.

nitrate medication should be readily available. All comorbidities and medication use should be recorded prior to the test.

\section{Patient preparation}

Patients should wear comfortable clothing and appropriate shoes for walking. Patients should use their usual walking aids during the test and this should be documented on the assessment form. Patients should not have exercised vigorously within $2 \mathrm{~h}$ of beginning the test but should have taken their usual medications. All subsequent testing occasions should occur at about the same time of day to minimise intraday variability, including variability in self-paced tests (6MWT) associated with bronchodilator use [109]. A warm-up is not permitted prior to commencing the test, neither is a shortened version of the test. If respiratory function tests are to be performed on the same day, this should occur prior to exercise testing, to avoid the confounding effects of exercise on these measures. The patients should then rest for at least 15 min before commencing an exercise test.

\section{Use of oxygen}

If a patient is on long-term oxygen therapy, oxygen should be given at their standard flow rate or as directed by a physician or a protocol. For any test where the outcome is distance, oxygen flow should be held constant throughout the test. If the purpose of the exercise test is to compare distance walked between tests, any subsequent test should be performed using the same oxygen conditions, in order to make a valid comparison between testing occasions. If oxygen supplementation is needed during the walks and serial tests are planned, then oxygen should be delivered in the same manner (flow rate and delivery device) for all subsequent walks. If the flow rate must be increased for subsequent visits due to worsening gas exchange, this should be noted on the worksheet and considered during interpretation of any changes in performance.

The type of oxygen delivery device should also be noted on the report: for instance, whether the subject carried liquid oxygen or pushed or pulled an oxygen tank, and whether the delivery was pulsed or continuous. Assessors should avoid transportation of the oxygen source where possible; however, if the subject is not able to control/carry/manage their own oxygen cylinder, the assessor should try to walk slightly behind the subject to avoid setting the walking pace. It should be clearly documented how the assessor has assisted with the transport of the oxygen, so any subsequent walk tests with the same subject can be performed in the same manner. Oxygen is not to be titrated during any of the tests where distance is a measured outcome. If oxygen titration is desired, this should be done during a separate test. 


\section{TABLE 5 Standardised instructions for the 6-min walk test}

The aim of this test is to walk as far as possible for 6 minutes. You will walk along this hallway between the markers, as many times as you can in 6 minutes.

I will let you know as each minute goes past, and then at 6 minutes I will ask you to stop where you are. 6 minutes is a long time to walk, so you will be exerting yourself. You are permitted to slow down, to stop, and to rest as necessary, but please resume walking as soon as you are able.

Remember that the objective is to walk AS FAR AS POSSIBLE for 6 minutes, but don't run or jog.

Do you have any questions?

\section{Medications}

Patients should be instructed to take all usual medications. The type of medication, dose and number of hours it was taken before the test should be noted. Significant improvement in the distance walked or the dyspnoea scale after administration of bronchodilators has been demonstrated in patients with COPD [93-96], although this may be small and clinically insignificant for the 6MWT [95, 110].

\section{Measurements}

Patients should rest in a chair, located near the starting position, before the test starts. Absolute and relative contraindications should be checked for prior to test commencement (table 4). The following measurements should be obtained at rest: $\mathrm{SpO}_{2}$ and $\mathrm{HR}$ from pulse oximetry, baseline dyspnoea and fatigue using a reproducible scale, and systemic blood pressure, if not recently documented [108].

\section{Immediately prior to the test}

The assessor should provide standardised instructions, either verbally for the 6MWT (table 5) or from the ISWT or ESWT audio recording. The patient should be positioned at the starting line. For the $6 \mathrm{MWT}$, the timer should be started as soon as the patient starts to walk. The recorded instructions of the ISWT and ESWT will prompt the individual to start.

A pulse oximeter should be used for continuous measurement of $\mathrm{SpO}_{2}$ and $\mathrm{HR}$. The assessor should not "pace" the patient during the test, but should walk behind such that measures of nadir $\mathrm{SpO}_{2}$ and end-test HR can be recorded without influencing the patient's movement. The assessor should ensure that the probe is placed such that a quality signal is obtained.

\section{Immediately on test cessation}

The $\mathrm{SpO}_{2}$ and pulse rate should be recorded from the oximeter, and the patient should be asked to rate their dyspnoea and subjective fatigue on the standardised scale. In addition, it is important to understand the reason for test termination/limitation, so patients should be asked why they could not walk any further. It is common for patients to report either dyspnoea or leg fatigue as the primary reason for a restricted performance.

\section{Reasons for the assessor to stop an exercise test}

In some individuals, profound desaturation $\left(\mathrm{SpO}_{2}<80 \%\right)$ may occur during a walking-based exercise test. Use of a 6MWT protocol that directs test cessation when $\mathrm{SpO}_{2}$ falls to $<80 \%$ is associated with an extremely low rate of adverse events [35]; this has not been reported for the ISWT or ESWT. Few data are available to determine the risk if the test is not stopped when $\mathrm{SpO}_{2}$ falls below $80 \%$. Ceasing a test when $\mathrm{SpO}_{2}$ falls to $<80 \%$ is also consistent with the recommendations for incremental exercise testing [108]. If $\mathrm{SpO}_{2}$ recovers to $\geqslant 85 \%$ during the $6 \mathrm{MWT}$, the patient may be asked to recommence walking.

Other reasons for test cessation include chest pain, intolerable dyspnoea, leg cramps, staggering, diaphoresis, and a pale or ashen appearance. If a test is stopped for any of these reasons, the patient should sit or lie supine as appropriate. The following should be obtained based on the judgment of the assessor: blood pressure, pulse rate, $\mathrm{SpO}_{2}$ and a physician evaluation. Oxygen should be administered as appropriate.

\section{Test repetition}

To establish a stable baseline for the 6MWT and ISWT so that change over time can be detected, two tests must be completed. These can be performed on the same day but there must be an interval between tests of at least $30 \mathrm{~min}$ and measures of $\mathrm{HR}$ and $\mathrm{SpO}_{2}$ must have returned to baseline prior to the second test.

\section{Quality assurance}

Consistency of testing procedures and conditions is critical to ensure that results are of good quality. It is important that all assessors are familiar with the test procedures, as the test requires clear processes to be followed. 


\section{TABLE 6 Standardised encouragement for the 6-min walk test}

$\begin{array}{ll}\mathbf{1} \text { min } & \text { You are doing well. You have } 5 \text { minutes to go. } \\ \mathbf{2} \text { min } & \text { Keep up the good work. You have } 4 \text { minutes to go. } \\ \mathbf{3} \text { min } & \text { You are doing well. You are halfway. } \\ \mathbf{4} \text { min } & \text { Keep up the good work. You have only } 2 \text { minutes left. } \\ \mathbf{5} \text { min } & \text { You are doing well. You have only } 1 \text { minute to go. } \\ \mathbf{6} \text { min } & \text { Please stop where you are. } \\ \text { If the patient stops during the test, every } & \text { Please resume walking whenever you feel able. } \\ \mathbf{3 0} \text { s once } \mathrm{SpO}_{\mathbf{2}} \text { is } \geqslant 85 \% & \end{array}$

$\mathrm{SpO}_{2}$ : arterial oxygen saturation measured by pulse oximetry.

For the ISWT and ESWT, the assessor must be able to walk at exactly the first speed of walking to pace the patient; this is particularly important for patients with a higher functional capacity where their natural speed of walking is much faster than the very slow pace required on the first level of the ISWT. All assessors should have their performance peer reviewed to ensure that performance of the tests is in accordance with the Technical Standard. Ideally, quality assurance testing should require the assessor to conduct the test on participants with a range of functional exercise capacity.

It must be ensured that the pulse oximeter is operating according to the manufacturer's instructions and that the track is accurately measured. The effects of any changes in testing scenario (e.g. location, equipment) should be evaluated to assess their effect on test outcomes. Any changes in procedures should be reviewed and approved by the programme director. Consistent documentation must be ensured for every test and a regular audit should be considered, to ensure that important procedures have been followed (e.g. recording of appropriate physiological signals at baseline and end-test, documentation of nadir $\mathrm{SpO}_{2}$, endtest $\mathrm{HR}$ and $6 \mathrm{MWD}$, consistent use of oxygen, appropriate test repetition).

\section{Testing protocol for 6MWT}

\section{Course}

The 6MWT should be performed along a flat, straight course with a hard surface with little pedestrian traffic. It is recommended that the walking course be $30 \mathrm{~m}$ or more in length, to be consistent with the courses on which reference equations have been generated [1]. The ends of the course should be marked such that they are easily visible to patients.

\section{Conduct}

The patient should be encouraged every $60 \mathrm{~s}$ using the standard phrases (table 6). Other words of encouragement and other nonverbal prompts should not be used. If the patient stops walking during the test, the timer must not be stopped. The patient should be allowed to rest while sitting or standing, as they prefer. While the patient is stopped, standardised encouragement should be provided every $30 \mathrm{~s}$ (table 6). The time that the patient stopped and the time that walking is recommenced should be recorded.

\section{Recording performance of the 6MWT}

The primary outcome to be reported is GMWD. The number of laps and any additional distance covered (the number of metres or feet in the final partial lap) should be recorded. The total distance walked is calculated, rounding to the nearest metre or foot. If the patient stopped during the test, the total time stopped, the number of stops and the average walking speed over the $6 \mathrm{~min}$ are also reported [111]. In patients who cannot walk for $6 \mathrm{~min}$, this may provide alternative metrics for detecting change over time [111] and may facilitate exercise prescription [112]. It is optional to report the 6MWD as a percentage of predicted. If the \% predicted 6MWD is reported, the reference equations used should be stated. Lowest $\mathrm{SpO}_{2}$, end-test $\mathrm{HR}$ and symptom scores obtained before and after the test should also be reported. A sample recording form can be found in the online supplementary material (table S1).

\section{Testing protocol for ISWT}

Course

The course is $10 \mathrm{~m}$ in length and is identified for the patient by two cones with an inset of $0.5 \mathrm{~m}$ from either end (fig. 1), thus avoiding abrupt changes in direction [113].

At the beginning of the test the instructions are played to the patient from an audio recording (table 7). Once the instructions have been played, and it is confirmed that the patient has understood the task, the 
FIGURE 1 Course layout for the incremental and endurance shuttle walk tests. Cones are inset $0.5 \mathrm{~m}$ from either end to avoid abrupt changes in direction.

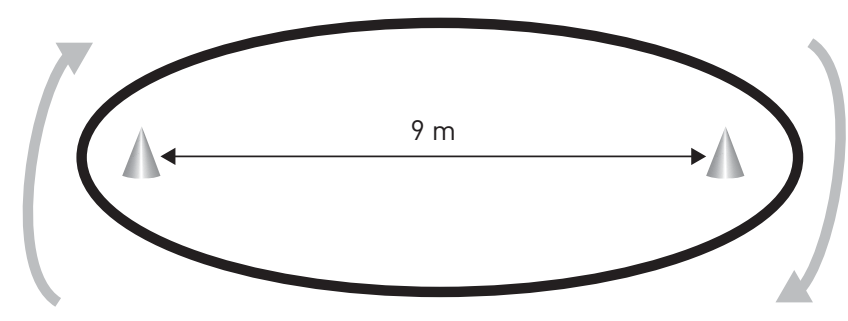

patient is positioned at one end of the course in preparation to start the test. The speed at which the patient should walk is directed by an audio signal and cannot be overridden. There is a triple bleep indicating the test has started: at this point the participant commences walking and the timer is activated.

\section{Conduct of the test}

It is important for the assessor to watch the patient but also to ensure they keep count of the number of lengths as the subject completes them, throughout the duration of the test. It is advisable to time the performance as an additional measure, to confirm manual recording of the number of shuttles completed. As the speed of walking increases every minute, indicated by a triple bleep [113], the patient should be advised: "You now need to increase your speed of walking." During the test, only one verbal cue can be used to encourage the patient to pick up their speed: "You need to increase your speed to keep up with the test."

\section{Termination of the test}

The test is terminated when either 1) the patient indicates that they are unable to continue, 2) the operator determines that the patient is not fit to continue, or 3) the operator assesses that the patient was unable to sustain the speed and cover the distance to the cone prior to the beep sounding [113].

\section{Operator termination of the test}

The operator will be required to terminate the test if the patient fails to reach the cone/marker in the time allowed [113]. This is defined as the patient being $>0.5 \mathrm{~m}$ away from the cone when the bleep sounds on a second successive $10-\mathrm{m}$ length. When the patient is just outside the $0.5-\mathrm{m}$ marker they are advised to increase their speed of walking; if the patient fails to do so then the test is terminated and the distance recorded.

The test should be discontinued by the operator if $\mathrm{SpO}_{2}$ falls below $80 \%$ as described in the ATS/American College of Chest Physicians (ACCP) statement on cardiopulmonary exercise testing [108].

\section{TABLE 7 Incremental and endurance shuttle walk test instructions}

\section{Incremental shuttle walk test instructions}

The object of the progressive shuttle walking test is to walk as long as possible, there and back along the 10-metre course, keeping to the speed indicated by the bleeps on the audio recording. You will hear these bleeps at regular intervals.

You should walk at a steady pace, aiming to turn around the cone at one end of the course when you hear the first bleep, and at the other end when you hear the next. At first, your walking speed will be very slow, but you will need to speed up at the end of each minute. Your aim should be to follow the set rhythm for as long as you can. Each single bleep signals the end of a shuttle and each triple bleep signals an increase in walking speed. You should stop walking only when you become too breathless to maintain the required speed or can no longer keep up with the set pace.

The test is maximal and progressive. In other words, it is easier at the start and harder at the end. The walking speed for the first minute is very slow. You have 20 seconds to complete each 10 -metre shuttle, so don't go too fast. The test will start in 15 seconds, so get ready at the start now. Level one starts with a triple bleep after the 4-second countdown.

\section{Endurance shuttle walk test instructions}

Walking test level ( 1 to 16 ). The instructions below are repeated for all 16 levels.

The walking speed for the first 2 minutes is fairly slow, so don't go too fast. The test will start in

10 seconds so get ready at the start now. The test starts with a triple bleep after a 4-second countdown. At the next triple bleep increase your walking speed. 
Participant termination of the test

The patient may terminate the test if they are unable to continue. In respiratory disease, the most common reason for terminating the test is excessive dyspnoea; however, other reasons may include fatigue (commonly leg fatigue) or pain (knee/hip/low back pain).

\section{Recording performance of the test}

The assessor should calculate the distance walked, in metres, including the last 10 -m length that was completed, and record this on the form available (see online supplementary material table S2).

\section{Testing protocol for ESWT}

Course

The test is conducted along the same course as described for the ISWT (fig. 1) [113].

\section{Conduct of the test}

Unlike the ISWT, the ESWT is not incremental and is performed at a constant speed, but there is a warmup period of $\sim 1.5 \mathrm{~min}$ [77]. At this point, there are standardised instructions for the participant played from the audio recording, advising the individual that at the next bleep the speed of walking will increase (table 7). This is the speed at which the test is performed. It is important to pre-define the speed at which the test is going to be conducted. This can be calculated from the ISWT: the speed may be taken from a pre-defined percentage of peak performance on the ISWT (e.g. 70-85\% estimated $V^{\prime} \mathrm{O}_{2}$ peak) [114] or a percentage of the peak speed achieved [115]. Once the instructions have been played to the patient, they should be directed to one end of the course. A triple bleep indicates that the test has started [77]. The initial stages of the test are at a slower speed and are a warm-up for the participant. After the warm-up period, the speed of walking increases; this is advised on the audio recording at the end of the warm-up period. The timer is started at the end of the warm-up period. Participants are then paced for the first two shuttles. During the test, only one verbal cue can be used to encourage the patient to pick up their speed: "You need to increase your speed to keep up with the test."

\section{Termination of the ESWT}

The procedure to terminate the test is as described for the ISWT [113].

\section{Recording performance of the test}

It is conventional for endurance performance to be recorded as time (in seconds). It is important that the speed of walking and time are recorded. A sample scoring sheet is included in the online supplementary material (table S2).

\section{Key changes in this updated Technical Standard}

Absolute and relative contraindications for field walking tests

Research published since 2002 has shown that, in adults with moderate chronic respiratory disease, the 6MWT and ISWT elicit a $V^{\prime} \mathrm{O}_{2}$ peak that is comparable to that on CPET [3-6, 8]. As a result, the absolute and relative contraindications for CPET have been adopted for field walking tests of exercise capacity.

\section{Continuous measurement of $\mathrm{SpO}_{2}$}

New data show both the importance of nadir $\mathrm{SpO}_{2}$ during the 6MWT as a marker of prognosis [38, 46, 116-119] and that end-test $\mathrm{SpO}_{2}$ frequently does not reflect this important value [40, 41]. These factors, together with improvements in pulse oximetry technology in managing motion artefact, have made it feasible and desirable to measure $\mathrm{SpO}_{2}$ continuously during field walking tests.

\section{MWT repetition}

There are now consistent and compelling data showing a learning effect for the 6MWD [1,9]. As a result, it is recommended that two 6MWTs are performed when the test is used to measure change over time.

\section{Addition of the ISWT and ESWT}

The ISWT and ESWT have emerged as robust and useful tests of exercise capacity in chronic respiratory disease [1]. The standard operating procedures for these tests are described here for the first time.

\section{Common testing procedures for field walking tests}

Testing procedures, such as test staffing, patient assessment, patient preparation, use of oxygen and medications, and quality assurance, are now consistent across the three field walking tests described in this Technical Standard. 


\section{Acknowledgements}

The authors' affiliations are as follows. Anne E. Holland: Dept of Physiotherapy, La Trobe University, Melbourne, Australia, Dept of Physiotherapy, Alfred Health, Melbourne, Australia, and Institute for Breathing and Sleep, Austin Health, Melbourne, Australia; Martijn A. Spruit: Dept of Research and Education, CIRO+ Centre of Expertise for Chronic Organ Failure, Horn, The Netherlands, and REVAL - Rehabilitation Research Center, BIOMED - Biomedical Research Institute, Faculty of Medicine and Life Sciences, Hasselt University, Diepenbeek, Belgium; Thierry Troosters and Carlos Augusto Camillo: Faculty of Kinesiology and Rehabilitation Sciences, Dept of Rehabilitation Sciences, Katholieke Universiteit Leuven, Leuven, Belgium; Milo A. Puhan: Institute for Social and Preventive Medicine, University of Zurich, Zurich, Switzerland; Véronique Pepin: Axe maladies chroniques, Centre de recherche de l'Hôpital du Sacré-Coeur de Montréal, Montreal, QC, Canada, and Dept of Exercise Science, Faculty of Arts and Science, Concordia University, Montreal, QC, Canada; Didier Saey: Centre de recherche, Institut Universitaire de cardiologie et de pneumologie de Québec, Québec, QC, Canada, and Faculté de médecine, Université Laval, Québec, QC, Canada; Meredith C. McCormack: Johns Hopkins University, Pulmonary and Critical Care Medicine, Baltimore, MD, USA; Brian W. Carlin: Drexel University School of Medicine, Pittsburgh, PA, USA, and Sleep Medicine and Lung Health Consultants, Pittsburgh, PA, USA; Frank C. Sciurba: University of Pittsburgh School of Medicine, Pittsburgh, PA, USA; Fabio Pitta and Nidia A. Hernandes: Laboratory of Research in Respiratory Physiotherapy, Dept of Physiotherapy, Universidade Estadual de Londrina, Londrina, Brazil; Jack Wanger: ATS Proficiency Standards for Pulmonary Function Laboratories Committee, Rochester, MN, USA; Neil MacIntyre: Duke University, Durham, NC, USA; David A. Kaminsky: University of Vermont College of Medicine, Burlington, VT, USA; Bruce H. Culver: Pulmonary and Critical Care Medicine, University of Washington, Seattle, WA, USA; Susan M. Revill: Clinical Diagnostix Ltd, Derby, UK; Vasileios Andrianopoulos: Dept of Research and Education, CIRO+ Centre of Expertise for Chronic Organ Failure, Horn, The Netherlands; Katy E. Mitchell: Centre for Exercise and Rehabilitation Science, University Hospitals of Leicester NHS Trust, Leicester, UK; Annemarie L. Lee: Dept of Physiotherapy, Alfred Health, Melbourne, Australia, and Institute for Breathing and Sleep, Austin Health, Melbourne, Australia; Catherine J. Hill: Institute for Breathing and Sleep, Austin Health, Melbourne, Australia, and Dept of Physiotherapy, Austin Health, Melbourne, Australia; Sally J. Singh: Centre for Exercise and Rehabilitation Science, University Hospitals of Leicester NHS Trust, Leicester, UK.

\section{References}

1 Singh SJ, Puhan MA, Andrianopoulos V, et al. An official systematic review of the European Respiratory Society/ American Thoracic Society: measurement properties of field walking tests in chronic respiratory disease. Eur Respir $J$ 2014; 44: 1447-1478.

2 ATS Committee on Proficiency Standards for Clinical Pulmonary Function Laboratories. ATS statement: guidelines for the six-minute walk test. Am J Respir Crit Care Med 2002; 166: 111-117.

3 Troosters T, Vilaro J, Rabinovich R, et al. Physiological responses to the 6-min walk test in patients with chronic obstructive pulmonary disease. Eur Respir J 2002; 20: 564-569.

4 Satake M, Shioya T, Takahashi H, et al. Ventilatory responses to six-minute walk test, incremental shuttle walking test, and cycle ergometer test in patients with chronic obstructive pulmonary disease. Biomed Res 2003; 24: 309-316.

5 Luxton N, Alison JA, Wu J, et al. Relationship between field walking tests and incremental cycle ergometry in COPD. Respirology 2008; 13: 856-862.

6 Hill K, Dolmage TE, Woon L, et al. Comparing peak and submaximal cardiorespiratory responses during field walking tests with incremental cycle ergometry in COPD. Respirology 2012; 17: 278-284.

7 Deboeck G, Niset G, Vachiery JL, et al. Physiological response to the six-minute walk test in pulmonary arterial hypertension. Eur Respir J 2005; 26: 667-672.

8 Blanco I, Villaquirán C, Valera JL, et al. Consumo maximo de oxigeno durante la prueba de marcha de 6 minutos en la enfermedad pulmonar intersticial difusa y en la hipertension pulmonar [Peak oxygen uptake during the sixminute walk test in diffuse interstitial lung disease and pulmonary hypertension]. Arch Bronconeumol 2010; 46: $122-128$.

9 Hernandes NA, Wouters EF, Meijer K, et al. Reproducibility of 6-minute walking test in patients with COPD. Eur Respir J 2011; 38: 261-267.

10 Spencer LM, Alison JA, McKeough ZJ. Six-minute walk test as an outcome measure: are two six-minute walk tests necessary immediately after pulmonary rehabilitation and at three-month follow-up? Am J Phys Med Rehabil 2008; 87: 224-228.

11 Chandra D, Kulkarni HS, Sciurba F. Learning from the learning effect in the six-minute-walk test. Am J Respir Crit Care Med 2012; 185: 684.

12 Cote CG, Pinto-Plata VM, Marin JM, et al. The modified BODE index: validation with mortality in COPD. Eur Respir J 2008; 32: 1269-1274.

13 Spruit MA, Polkey MI, Celli B, et al. Predicting outcomes from 6-minute walk distance in chronic obstructive pulmonary disease. J Am Med Dir Assoc 2012; 13: 291-297.

14 Stevens D, Elpern E, Sharma K, et al. Comparison of hallway and treadmill six-minute walk tests. Am J Respir Crit Care Med 1999; 160: 1540-1543.

15 de Almeida FG, Victor EG, Rizzo JA. Hallway versus treadmill 6-minute-walk tests in patients with chronic obstructive pulmonary disease. Respir Care 2009; 54: 1712-1716.

16 Brooks D, Solway S, Weinacht K, et al. Comparison between an indoor and an outdoor 6-minute walk test among individuals with chronic obstructive pulmonary disease. Arch Phys Med Rehabil 2003; 84: 873-876.

17 Bansal V, Hill K, Dolmage TE, et al. Modifying track layout from straight to circular has a modest effect on the 6-min walk distance. Chest 2008; 133: 1155-1160.

18 Sciurba F, Criner GJ, Lee SM, et al. Six-minute walk distance in chronic obstructive pulmonary disease: reproducibility and effect of walking course layout and length. Am J Respir Crit Care Med 2003; 167: 1522-1527.

19 Beekman E, Mesters I, Hendriks EJ, et al. Course length of 30 metres versus 10 metres has a significant influence on six-minute walk distance in patients with COPD: an experimental crossover study. J Physiother 2013; 59: 169-176.

20 Gupta R, Goldstein R, Brooks D. The acute effects of a rollator in individuals with COPD. J Cardiopulm Rehabil 2006; 26: 107-111. 
21 Honeyman P, Barr P, Stubbing DG. Effect of a walking aid on disability, oxygenation, and breathlessness in patients with chronic airflow limitation. J Cardiopulm Rehabil 1996; 16: 63-67.

22 Probst VS, Troosters T, Coosemans I, et al. Mechanisms of improvement in exercise capacity using a rollator in patients with COPD. Chest 2004; 126: 1102-1107.

23 Roomi J, Yohannes AM, Connolly MJ. The effect of walking aids on exercise capacity and oxygenation in elderly patients with chronic obstructive pulmonary disease. Age Ageing 1998; 27: 703-706.

24 Solway S, Brooks D, Lau L, et al. The short-term effect of a rollator on functional exercise capacity among individuals with severe COPD. Chest 2002; 122: 56-65.

25 Vaes AW, Annegarn J, Meijer K, et al. The effects of a "new" walking aid on exercise performance in patients with COPD: a randomized crossover trial. Chest 2012; 141: 1224-1232.

26 Davidson AC, Leach R, George RJ, et al. Supplemental oxygen and exercise ability in chronic obstructive airways disease. Thorax 1988; 43: 965-971.

27 Fujimoto K, Matsuzawa Y, Yamaguchi S, et al. Benefits of oxygen on exercise performance and pulmonary hemodynamics in patients with COPD with mild hypoxemia. Chest 2002; 122: 457-463.

28 Rooyackers JM, Dekhuijzen PN, Van Herwaarden CL, et al. Training with supplemental oxygen in patients with COPD and hypoxaemia at peak exercise. Eur Respir J 1997; 10: 1278-1284.

29 Jolly EC, Di Boscio V, Aguirre L, et al. Effects of supplemental oxygen during activity in patients with advanced COPD without severe resting hypoxemia. Chest 2001; 120: 437-443.

30 McDonald CF, Blyth CM, Lazarus MD, et al. Exertional oxygen of limited benefit in patients with chronic obstructive pulmonary disease and mild hypoxemia. Am J Respir Crit Care Med 1995; 152: 1616-1619.

31 Crisafulli E, Beneventi C, Bortolotti V, et al. Energy expenditure at rest and during walking in patients with chronic respiratory failure: a prospective two-phase case-control study. PLoS One 2011; 6: e23770.

32 Woodcock AA, Gross ER, Geddes DM. Oxygen relieves breathlessness in "pink puffers". Lancet 1981; 1: 907-909.

33 Guyatt GH, Pugsley SO, Sullivan MJ, et al. Effect of encouragement on walking test performance. Thorax 1984; 39: 818-822.

34 Weir NA, Brown AW, Shlobin OA, et al. The influence of alternative instruction on 6-min walk test distance. Chest 2013; 144: 1900-1905.

35 Jenkins S, Čečins N. Six-minute walk test: observed adverse events and oxygen desaturation in a large cohort of patients with chronic lung disease. Intern Med J 2011; 41: 416-422.

36 Park JH, Jegal Y, Shim TS, et al. Hypoxemia and arrhythmia during daily activities and six-minute walk test in fibrotic interstitial lung diseases. J Korean Med Sci 2011; 26: 372-378.

37 van Gestel AJ, Clarenbach CF, Stöwhas AC, et al. Prevalence and prediction of exercise-induced oxygen desaturation in patients with chronic obstructive pulmonary disease. Respiration 2012; 84: 353-359.

38 Casanova C, Cote C, Marin JM, et al. Distance and oxygen desaturation during the 6-min walk test as predictors of long-term mortality in patients with COPD. Chest 2008; 134: 746-752.

39 Poulain M, Durand F, Palomba B, et al. 6-minute walk testing is more sensitive than maximal incremental cycle testing for detecting oxygen desaturation in patients with COPD. Chest 2003; 123: 1401-1407.

40 Chuang ML, Lin IF, Chen SP. Kinetics of changes in oxyhemoglobin saturation during walking and cycling tests in COPD. Respir Care 2014; 59: 353-362.

41 Fiore C, Lee A, McDonald C, et al. Should oxyhaemoglobin saturation be monitored continuously during the 6-minute walk test? Chron Respir Dis 2011; 8: 181-184.

42 Lettieri CJ, Nathan SD, Browning RF, et al. The distance-saturation product predicts mortality in idiopathic pulmonary fibrosis. Respir Med 2006; 100: 1734-1741.

43 Modrykamien AM, Gudavalli R, McCarthy K, et al. Echocardiography, 6-minute walk distance, and distancesaturation product as predictors of pulmonary arterial hypertension in idiopathic pulmonary fibrosis. Respir Care 2010; 55: 584-588.

44 Alhamad EH, Shaik SA, Idrees MM, et al. Outcome measures of the 6 minute walk test: relationships with physiologic and computed tomography findings in patients with sarcoidosis. BMC Pulm Med 2010; 10: 42.

45 Bourbonnais JM, Malaisamy S, Dalal BD, et al. Distance saturation product predicts health-related quality of life among sarcoidosis patients. Health Qual Life Outcomes 2012; 10: 67.

46 Flaherty KR, Andrei AC, Murray S, et al. Idiopathic pulmonary fibrosis: prognostic value of changes in physiology and six-minute-walk test. Am J Respir Crit Care Med 2006; 174: 803-809.

47 Pimenta SP, Rocha RB, Baldi BG, et al. Desaturation-distance ratio: a new concept for a functional assessment of interstitial lung diseases. Clinics 2010; 65: 841-846.

48 Mandrusiak A, Maurer C, MacDonald J, et al. Functional capacity tests in young people with cystic fibrosis. $N$ Z J Physiother 2009; 37: 13-16.

49 Swigris JJ, Swick J, Wamboldt FS, et al. Heart rate recovery after 6-min walk test predicts survival in patients with idiopathic pulmonary fibrosis. Chest 2009; 136: 841-848.

50 Ramos RP, Arakaki JS, Barbosa P, et al. Heart rate recovery in pulmonary arterial hypertension: relationship with exercise capacity and prognosis. Am Heart J 2012; 163: 580-588.

51 Minai OA, Gudavalli R, Mummadi S, et al. Heart rate recovery predicts clinical worsening in patients with pulmonary arterial hypertension. Am J Respir Crit Care Med 2012; 185: 400-408.

52 Inal-Ince D, Savci S, Coplu L, et al. Functional capacity in severe chronic obstructive pulmonary disease. Saudi Med J 2005; 26: 84-89.

53 van Stel HF, Bogaard JM, Rijssenbeek-Nouwens LH, et al. Multivariable assessment of the 6-min walking test in patients with chronic obstructive pulmonary disease. Am J Respir Crit Care Med 2001; 163: 1567-1571.

54 Alhamad EH. The six-minute walk test in patients with pulmonary sarcoidosis. Ann Thorac Med 2009; 4: 60-64.

55 Spruit MA, Watkins ML, Edwards LD, et al. Determinants of poor 6-min walking distance in patients with COPD: the ECLIPSE cohort. Respir Med 2010; 104: 849-857.

56 Callens E, Graba S, Gillet-Juvin K, et al. Measurement of dynamic hyperinflation after a 6-minute walk test in patients with COPD. Chest 2009; 136: 1466-1472.

57 Nishiyama O, Taniguchi $\mathrm{H}$, Kondoh $\mathrm{Y}$, et al. Dyspnoea at 6-min walk test in idiopathic pulmonary fibrosis: comparison with COPD. Respir Med 2007; 101: 833-838. 
58 Gallego MC, Samaniego J, Alonso J, et al. Disnea en la EPOC: relacion de la escala MRC con la disnea inducida en las pruebas de marcha y de ejercicio cardiopulmonar maximo [Dyspnea in COPD: relation to the MRC scale with dyspnea induced by walking and cardiopulmonary stress testing]. Arch Bronconeumol 2002; 38: 112-116.

59 Borg GA. Psychophysical bases of perceived exertion. Med Sci Sports Exerc 1982; 14: 377-381.

60 Annegarn J, Spruit MA, Savelberg HH, et al. Differences in walking pattern during 6-min walk test between patients with COPD and healthy subjects. PLoS One 2012; 7: e37329.

61 Ziegler B, Rovedder PM, Oliveira CL, et al. Repeatability of the 6-minute walk test in adolescents and adults with cystic fibrosis. Respir Care 2010; 55: 1020-1025.

62 Gagnon P, Maltais F, Bouyer L, et al. Distal leg muscle function in patients with COPD. COPD 2013; 10: $235-242$.

63 Chuang ML, Lin IF, Wasserman K. The body weight-walking distance product as related to lung function, anaerobic threshold and peak $V^{\prime} \mathrm{O}_{2}$ in COPD patients. Respir Med 2001; 95: 618-626.

64 Carter R, Holiday DB, Nwasuruba C, et al. 6-minute walk work for assessment of functional capacity in patients with COPD. Chest 2003; 123: 1408-1415.

65 Puhan MA, Mador MJ, Held U, et al. Interpretation of treatment changes in 6-minute walk distance in patients with COPD. Eur Respir J 2008; 32: 637-643.

66 Puhan MA, Chandra D, Mosenifar Z, et al. The minimal important difference of exercise tests in severe COPD. Eur Respir J 2011; 37: 784-790.

67 Polkey MI, Spruit MA, Edwards LD, et al. Six-minute-walk test in chronic obstructive pulmonary disease: minimal clinically important difference for death or hospitalization. Am J Respir Crit Care Med 2013; 187: 382-386.

68 Holland AE, Hill CJ, Conron M, et al. Small changes in six-minute walk distance are important in diffuse parenchymal lung disease. Respir Med 2009; 103: 1430-1435.

69 Holland AE, Hill CJ, Rasekaba T, et al. Updating the minimal important difference for six-minute walk distance in patients with chronic obstructive pulmonary disease. Arch Phys Med Rehabil 2010; 91: 221-225.

70 Lacasse Y, Goldstein R, Lasserson TJ, et al. Pulmonary rehabilitation for chronic obstructive pulmonary disease. Cochrane Database Syst Rev 2006; 4: CD003793.

71 Puhan M, Scharplatz M, Troosters T, et al. Pulmonary rehabilitation following exacerbations of chronic obstructive pulmonary disease. Cochrane Database Syst Rev 2009; 1: CD005305.

72 Holland A, Hill C. Physical training for interstitial lung disease. Cochrane Database Syst Rev 2008; 4: CD006322.

73 Liu C, Chen J, Gao Y, et al. Endothelin receptor antagonists for pulmonary arterial hypertension. Cochrane Database Syst Rev 2009; 3: CD004434.

74 Britto RR, Probst VS, de Andrade AF, et al. Reference equations for the six-minute walk distance based on a Brazilian multicenter study. Braz J Phys Ther 2013; 17: 556-563.

75 Palange $\mathrm{P}$, Forte S, Onorati $\mathrm{P}$, et al. Ventilatory and metabolic adaptations to walking and cycling in patients with COPD. J Appl Physiol 2000; 88: 1715-1720.

76 Zainuldin R, Mackey MG, Alison JA. Prescription of walking exercise intensity from the incremental shuttle walk test in people with chronic obstructive pulmonary disease. Am J Phys Med Rehabil 2012; 91: 592-600.

77 Revill SM, Morgan MD, Singh SJ, et al. The endurance shuttle walk: a new field test for the assessment of endurance capacity in chronic obstructive pulmonary disease. Thorax 1999; 54: 213-222.

78 Campo LA, Chilingaryan G, Berg K, et al. Validity and reliability of the modified shuttle walk test in patients with chronic obstructive pulmonary disease. Arch Phys Med Rehabil 2006; 87: 918-922.

79 Eiser N, Willsher D, Doré CJ. Reliability, repeatability and sensitivity to change of externally and self-paced walking tests in COPD patients. Respir Med 2003; 97: 407-414.

80 Dyer F, Marriner P, Cheema K, et al. Is a practice incremental shuttle walk test really necessary? Chron Respir Dis 2011; 8: 201-205.

81 McKeough ZJ, Leung RW, Alison JA. Shuttle walk tests as outcome measures: are two incremental shuttle walk tests and two endurance shuttle walk tests necessary? Am J Phys Med Rehabil 2011; 90: 35-39.

82 Revill SM, Williams J, Sewell L, et al. Within-day repeatability of the endurance shuttle walk test. Physiotherapy 2009; 95: 140-143.

83 Emtner MI, Arnardottir HR, Hallin R, et al. Walking distance is a predictor of exacerbations in patients with chronic obstructive pulmonary disease. Respir Med 2007; 101: 1037-1040.

84 Ringbaek T, Martinez G, Brøndum E, et al. Shuttle walking test as predictor of survival in chronic obstructive pulmonary disease patients enrolled in a rehabilitation program. J Cardiopulm Rehabil Prev 2010; 30: 409-414.

85 Williams JE, Green RH, Warrington V, et al. Development of the i-BODE: validation of the incremental shuttle walking test within the BODE index. Respir Med 2012; 106: 390-396.

86 Sandland CJ, Morgan MD, Singh SJ. Detecting oxygen desaturation in patients with COPD: incremental versus endurance shuttle walking. Respir Med 2008; 102: 1148-1152.

87 Laude EA, Duffy NC, Baveystock C, et al. The effect of helium and oxygen on exercise performance in chronic obstructive pulmonary disease: a randomized crossover trial. Am J Respir Crit Care Med 2006; 173: 865-870.

88 Morales FJ, Montemayor T, Martinez A. Shuttle versus six-minute walk test in the prediction of outcome in chronic heart failure. Int J Cardiol 2000; 76: 101-105.

89 Jolly K, Taylor RS, Lip GY, et al. Reproducibility and safety of the incremental shuttle walking test for cardiac rehabilitation. Int J Cardiol 2008; 125: 144-145.

90 Evans RA, Singh SJ, Collier R, et al. Generic, symptom based, exercise rehabilitation; integrating patients with COPD and heart failure. Respir Med 2010; 104: 1473-1481.

91 Singh SJ, Jones PW, Evans R, et al. Minimum clinically important improvement for the incremental shuttle walking test. Thorax 2008; 63: 775-777.

92 Dodd JW, Hogg L, Nolan J, et al. The COPD assessment test (CAT): response to pulmonary rehabilitation. A multicentre, prospective study. Thorax 2011; 66: 425-429.

93 Pepin V, Laviolette L, Brouillard C, et al. Significance of changes in endurance shuttle walking performance. Thorax 2011; 66: 115-120.

94 Pepin V, Saey D, Whittom F, et al. Walking versus cycling: sensitivity to bronchodilation in chronic obstructive pulmonary disease. Am J Respir Crit Care Med 2005; 172: 1517-1522.

95 Pepin V, Brodeur J, Lacasse Y, et al. Six-minute walking versus shuttle walking: responsiveness to bronchodilation in chronic obstructive pulmonary disease. Thorax 2007; 62: 291-298. 
96 Brouillard C, Pepin V, Milot J, et al. Endurance shuttle walking test: responsiveness to salmeterol in COPD. Eur Respir J 2008; 31: 579-584.

97 Dyer CA, Singh SJ, Stockley RA, et al. The incremental shuttle walking test in elderly people with chronic airflow limitation. Thorax 2002; 57: 34-38.

98 Eaton T, Young P, Nicol K, et al. The endurance shuttle walking test: a responsive measure in pulmonary rehabilitation for COPD patients. Chron Respir Dis 2006; 3: 3-9.

99 Leung RW, Alison JA, McKeough ZJ, et al. Ground walk training improves functional exercise capacity more than cycle training in people with chronic obstructive pulmonary disease (COPD): a randomised trial. J Physiother 2010; 56: $105-112$.

100 Revill SM, Noor MZ, Butcher G, et al. The endurance shuttle walk test: an alternative to the six-minute walk test for the assessment of ambulatory oxygen. Chron Respir Dis 2010; 7: 239-245.

101 Jürgensen SP, Antunes LC, Tanni SE, et al. The incremental shuttle walk test in older Brazilian adults. Respiration 2011; 81: 223-228.

102 Probst VS, Hernandes NA, Teixeira DC, et al. Reference values for the incremental shuttle walking test. Respir Med 2012; 106: 243-248.

103 Harrison SL, Greening NJ, Houchen-Wolloff L, et al. Age-specific normal values for the incremental shuttle walk test in a healthy British population. J Cardiopulm Rehabil Prev 2013; 33: 309-313.

104 Onorati P, Antonucci R, Valli G, et al. Non-invasive evaluation of gas exchange during a shuttle walking test $v s$ a 6-min walking test to assess exercise tolerance in COPD patients. Eur J Appl Physiol 2003; 89: 331-336.

105 Turner SE, Eastwood PR, Cecins NM, et al. Physiologic responses to incremental and self-paced exercise in COPD: a comparison of three tests. Chest 2004; 126: 766-773.

106 Casas A, Vilaro J, Rabinovich R, et al. Encouraged 6-min walking test indicates maximum sustainable exercise in COPD patients. Chest 2005; 128: 55-61.

107 McNamara RJ, McKeough ZJ, McKenzie DK, et al. Water-based exercise in COPD with physical comorbidities: a randomised controlled trial. Eur Respir J 2013; 41: 1284-1291.

108 American Thoracic Society, American College of Chest Physicians. ATS/ACCP Statement on cardiopulmonary exercise testing. Am J Respir Crit Care Med 2003; 167: 211-277.

109 Um SW, Yoo CG, Kim YW, et al. The combination of tiotropium and budesonide in the treatment of chronic obstructive pulmonary disease. J Korean Med Sci 2007; 22: 839-845.

110 Oga T, Nishimura K, Tsukino M, et al. The effects of oxitropium bromide on exercise performance in patients with stable chronic obstructive pulmonary disease. A comparison of three different exercise tests. Am J Respir Crit Care Med 2000; 161: 1897-1901.

111 Hallstrand TS, Boitano LJ, Johnson WC, et al. The timed walk test as a measure of severity and survival in idiopathic pulmonary fibrosis. Eur Respir J 2005; 25: 96-103.

112 Spruit MA, Singh SJ, Garvey C, et al. An official American Thoracic Society/European Respiratory Society statement: key concepts and advances in pulmonary rehabilitation. Am J Respir Crit Care Med 2013; 188: e13-e64.

113 Singh SJ, Morgan MD, Scott S, et al. Development of a shuttle walking test of disability in patients with chronic airways obstruction. Thorax 1992; 47: 1019-1024.

114 Singh SJ, Morgan MD, Hardman AE, et al. Comparison of oxygen uptake during a conventional treadmill test and the shuttle walking test in chronic airflow limitation. Eur Respir J 1994; 7: 2016-2020.

115 Hill K, Dolmage TE, Woon L, et al. A simple method to derive speed for the endurance shuttle walk test. Respir Med 2012; 106: 1665-1670.

116 Takigawa N, Tada A, Soda R, et al. Distance and oxygen desaturation in 6-min walk test predict prognosis in COPD patients. Respir Med 2007; 101: 561-567.

117 Lama VN, Flaherty KR, Toews GB, et al. Prognostic value of desaturation during a 6-minute walk test in idiopathic interstitial pneumonia. Am J Respir Crit Care Med 2003; 168: 1084-1090.

118 Paciocco G, Martinez FJ, Bossone E, et al. Oxygen desaturation on the six-minute walk test and mortality in untreated primary pulmonary hypertension. Eur Respir J 2001; 17: 647-652.

119 Villalba WO, Sampaio-Barros PD, Pereira MC, et al. Six-minute walk test for the evaluation of pulmonary disease severity in scleroderma patients. Chest 2007; 131: 217-222. 